\title{
Metodologías ágiles: un análisis de los desafíos organizacionales para su implementación
}

\author{
Agile Methodologies: An Analysis of the Organizational Challenges for their \\ Implementation
}

\author{
Metodologias ágeis: Uma análise dos desafios organizacionais para a sua \\ implementação
}

\author{
Fernando Flores-Cerna ${ }^{1}$ \\ Víctor-Manuel Sanhueza-Salazar² \\ Héctor-Moisés Valdés-González ${ }^{3}$ \\ Lorenzo Reyes-Bozo ${ }^{4}$
}

Recibido: agosto de 2021

Aceptado: octubre de 2021

Para citar este artículo: Flores-Cerna, F., Sanhueza-Salazar, V. M., Valdés-González, H. M. y Reyes-Bozo, L. (2022). Metodologías ágiles: un análisis de los desafíos organizacionales para su implementación. Revista Científica, 43(1), 38-49. https://doi.org/10.14483/23448350.18332

\section{Resumen}

Este trabajo analiza las principales brechas que influyen en las PyMEs TI (pequeñas y medianas empresas de tecnología de la información) con metodología tradicional, al iniciar la implementación de metodologías ágiles para la gestión de proyectos. El objetivo de esta investigación es determinar las principales brechas existentes antes de implementar metodologías ágiles en una PyME TI con metodología tradicional para la gestión de proyectos. Para lograrlo se propone una aproximación cualitativa a las opiniones de tres gerentes, dos ingenieros de proyecto y seis analistas programadores de la empresa, basada en entrevistas semiestructuradas, considerando una muestra por conveniencia, para entender las dificultades existentes en la empresa de estudio, contrastando dicha realidad mediante encuestas dirigidas a 29 profesionales de áreas TI de otras organizaciones. Los resultados del análisis revelan que las principales brechas para la implementación de metodologías ágiles en la PyME son la cultura organizacional en desacuerdo con la agilidad, la falta de compromiso de la gerencia y la resistencia al cambio. Se concluye que la propuesta resulta ser efectiva para conocer las principales brechas a resolver antes de comenzar la implementación de una metodología ágil en una PyME TI, permitiendo alinear a la organización con la agilidad.

Palabras clave: agilidad; gestión de proyectos; metodología tradicional; objetivos estratégico; PyME; tecnologías de la información.

1. Universidad del Desarrollo. Santiago, Chile. karen.guzman@uptc.edu.co.

2. Universidad del Desarrollo. Santiago, Chile. marco.suarez@uptc.edu.co.

3. Universidad del Desarrollo. Santiago, Chile. hvaldes@udd.cl.

4. Universidad Autónoma de Chile. Santiago, Chile. lorenzo.reyes@uautonoma.cl. 


\section{Abstract}

The objective of this research is to determine the main existing gaps before implementing agile methodologies in IT-SMEs (Information Technology - Small and Medium-Sized Enterprises) with traditional methodology for project management. To this effect, a qualitative approach to the opinions of three managers, two project engineers, and six programmer analysts of a company is proposed, which is based on semi-structured interviews, considering a convenience sample, in order to understand the existing difficulties in the company under study, contrasting this reality through surveys aimed at 29 IT professionals from other organizations. The results of the analysis reveal that the main gaps for the implementation of agile methodologies in SMEs are organizational culture at odds with agility, lack of commitment from management, and resistance to change. It is concluded that the proposal turns out to be effective for understanding the main gaps to be solved before starting the implementation of an agile methodology in an IT-SME, thus allowing the organization to be aligned with agility.

Keywords: agility; information technologies; project management; SMEs; strategic objectives; traditional methodology.

\section{Resumo}

Este artigo analisa as principais lacunas que influenciam as Pequenas e Médias Empresas (PMEs) de TI (Tecnologia da Informação) com metodologia tradicional, ao iniciar a implementação de metodologias ágeis de gestão de projetos. O objetivo desta pesquisa é determinar as principais lacunas existentes antes de implementar metodologias ágeis em uma PMEs de TI com metodologia tradicional para gerenciamento de projetos. Para tanto, é proposta uma abordagem qualitativa a partir das opiniões de 3 gestores, 2 engenheiros de projeto e 6 analistas programadores da empresa, com base em entrevistas semiestruturadas, considerando uma amostra de conveniência, para compreender as dificuldades existentes na empresa em estudo, contrastando essa realidade por meio de pesquisas dirigidas a 29 profissionais de TI de outras organizações. Os resultados da análise revelam que as principais lacunas para a implementação de metodologias ágeis nas PMEs são a cultura organizacional em desacordo com a agilidade, a falta de compromisso da gestão e a resistência à mudança. Conclui-se que a proposta mostra-se eficaz para conhecer as principais lacunas a resolver antes de iniciar a implantação de uma metodologia ágil em uma PMEs de TI, permitindo que a organização esteja alinhada com agilidade.

Palavras-chaves: agilidade; gestão de projetos; metodologia tradicional; objetivos estratégicos; PMEs; tecnologias de informação.

\section{Introducción}

Las metodologías tradicionales surgieron en la década de 1970, estas se fundamentan en la anticipación de todos los requerimientos del sistema, con el objetivo de realizar una planificación previa de cómo funcionará. Aquí los esfuerzos están dirigidos a obtener artefactos del proyecto a través de informes, documentos y diagramas completos y precisos (Nishijima y Dos Santos, 2013). Los métodos de desarrollo de software que surgieron de esta metodología — como el método en cascada- son ideales para equipos de proyectos con bajo rendimiento, ya que las tareas están estrictamente definidas y el progreso del proyecto es fácil de seguir ya que una fase comienza al terminar la anterior. Sin embargo, responder a mejoras o correcciones es difícil y costoso debido a que afectan a las etapas posteriores (Göksu, 2018), además, una documentación excesiva en las primeras etapas de un proyecto puede ser algo problemático y engañoso en un entorno dinámico (Collyer et al., 2010), ya que las inconsistencias en los requerimientos solo se pueden detectar en las fases de diseño y codificación.

Ante esto, en la década posterior surge el modelo espiral como una variante de gestión de riesgos del modelo en cascada, siendo el comienzo de los enfoques incrementales y el desarrollo de software basado en riesgos (Hohl et al., 2018). Estas décadas de desarrollo de software y prácticas marcan la antesala de lo que serían las metodologías 
ágiles, que fueron introducidas formalmente a la ingeniería de software a través del Manifiesto Ágil en 2001 (Beck et al., 2001) con un postulado de cuatro valores y doce principios fundamentales: personas e interacciones sobre procesos y herramientas, software funcionando sobre una documentación exhaustiva, colaboración con el cliente sobre la negociación contractual y responder ante el cambio sobre seguir un plan, orientándose a un flujo iterativo y a la entrega incremental de productos de software en iteraciones cortas (Patanakul, Henry y Leach, 2015).

Desde el origen del manifiesto ágil han surgido diversos estudios que analizan la metodología, como el propuesto por de Souza Bermejo et al. (2014), orientado a identificar los perfiles de organizaciones que producen software con principios ágiles y su éxito, afirmando que las organizaciones con las tasas de éxito más altas son las mismas que tienen altas capacidades en términos de equipo, cultura, comunicación con los clientes y relaciones con los colaboradores y externos. Pikkarainen et al. (2012) en su estudio identificaron los factores de éxito y las recomendaciones para una implementación ágil en las empresas de software, reconociendo la importancia de definir un modelo de proceso y otorgar a los desarrolladores la libertad de mejorar su propio proceso de desarrollo ágil durante la implementación.

Senapathi y Srinivasan (2012) centraron su estudio en el estado actual de diversas organizaciones después de adoptar metodologías ágiles. En su estudio a la BBCW (BBC Worldwide) que adoptó la metodología ágil Kanban en combinación con Scrum, evidenciaron mejoras en el rendimiento del equipo de desarrollo, en cuanto a los tiempos de entrega del software en un $37 \%$ y disminución de los defectos en un 24\%. En el caso de Stats NAZ (Statistics New Zealand) que realizó una migración desde metodología cascada a Scrum, se evidenció que antes de la migración los equipos enfrentaban dificultades en la priorización de requerimientos y tenían retrasos en la entrega al cliente, afectando la moral del equipo. Los autores concluyen que mientras más se asimilen las prácticas ágiles en la organización, mejores serán los resultados, y que factores como los de innovación ágil (ventaja relativa, compatibilidad), los sociológicos (experiencia, actitud, competencia técnica), los tecnológicos (prácticas ágiles, soporte, herramientas) y los organizacionales influyen en el uso efectivo de las metodologías ágiles. Esto tiene relación directa con el estudio realizado por PMI (2018), que indica que el $71 \%$ de las organizaciones ha aumentado su competitividad mediante la agilidad.

En el estudio presentado por Mohallel y Bass $\underline{(2019)}$, que analizó los principales desafíos durante el desarrollo de proyectos ágiles en PyMEs (pequeñas y medianas empresas), se evidenció que la planificación insuficiente, la presión constante, la falta de disponibilidad de tiempo en los equipos de desarrollo y la inadecuada estimación de recursos fueron los principales problemas durante la adopción, esto debido a la poca colaboración del equipo de desarrollo en las estimaciones al asumir que estas se sobreestiman para trabajar de forma relajada.

Respecto a la implementación de metodologías ágiles en micro, pequeñas y medianas empresas, Yepes, Pardo y Gómez (2015) concluyeron que los factores clave para que la agilidad falle dentro de una organización son la falta de entendimiento y empoderamiento en la metodología, además de la falta de compromiso de los clientes. Ante esto recomiendan que las organizaciones y los equipos de trabajo interioricen en su totalidad la metodología ágil, generando las modificaciones necesarias para adecuarse a las características particulares del entorno.

Las organizaciones dedicadas al desarrollo de proyectos de software comprenden que la gestión de proyectos es su principal competencia estratégica y que poseer procesos estandarizados conduce a mejores resultados. En esta línea, la administración y los gerentes de proyectos deben ser capaces de adaptar estos procesos constantemente para mantenerse competitivos en entornos cada vez más cambiantes, con el objetivo de reducir 
costos y tiempos de entrega, y mejorar la calidad, alejándose de las metodologías tradicionales que obligan a definir previamente todas las necesidades del cliente. Es por ello que actualmente el $97 \%$ de las organizaciones practica métodos de desarrollo ágiles, pero el 83\% aún está en una etapa de maduración respecto a las prácticas y un $78 \%$ indica que no todos los equipos en la organización las han adoptado (CollabNet VersionOne, 2019), evidenciando así que las organizaciones aún están en una etapa de maduración y que presentan desafíos en la etapa de adopción y en el uso de sus prácticas. Las organizaciones adoptan la agilidad porque creen en los valores y principios del manifiesto o porque consideran que es la mejor práctica (Hohl et al., 2018); sin embargo, muchos desarrolladores y gerentes adoptan la agilidad sin ser conscientes de la diversidad de principios y métodos ágiles, siendo vista Scrum como la única práctica ágil, interpretando la agilidad de forma errónea (Klünder et al., 2017), Ilevando a un aumento en la tasa de fracasos durante la etapa de adopción de una metodología ágil al no existir conocimiento previo sobre las principales brechas a abordar por las empresas.

Finalmente, y habiendo revisado las principales contribuciones que aportan o han aportado a la línea de trabajo, es posible indicar que una oportunidad de desarrollo se encuentra en el hecho de que no existe, para el caso de una PyME TI, suficiente información o certeza respecto de las brechas a superar antes de adoptar metodologías ágiles con el fin de apoyar una correcta etapa de adopción. Con base en lo anterior surge el siguiente cuestionamiento: ¿cómo es posible implementar la agilidad en una PyME TI que utiliza metodología tradicional para la gestión de proyectos?

Habiendo recorrido las bases teóricas fundamentales para este estudio, cabe mencionar que la principal motivación para realizarlo tiene relación con comprender las principales brechas que enfrenta una PyME TI con metodología tradicional al querer implementar metodologías ágiles, comparando cómo se relacionan estos desafíos con la realidad de otras organizaciones TI, con el propósito de verificar en qué medida la empresa de estudio se ajusta a la realidad nacional. Ante esto, se propone un estudio cualitativo de la percepción de los diferentes niveles jerárquicos de la compañía frente a una futura implementación ágil como método para la gestión de proyectos. En este sentido, este estudio contribuye a identificar las principales brechas a las que una organización con metodología tradicional se ve expuesta al momento de implementar metodologías ágiles para la gestión de proyectos, permitiendo abordarlas antes de comenzar la etapa de transición.

Entendido esto, el objetivo de este trabajo es determinar las principales brechas a resolver antes de implementar metodologías ágiles para la gestión de proyectos en una PyME TI con metodología tradicional.

\section{Metología}

\section{Paradigma y diseño}

La investigación propone la utilización de una metodología cualitativa, basada en entrevistas semiestructuradas, considerando una muestra por conveniencia (Rodríguez y Valldeoriola, 2009), efectuada a los diferentes niveles jerárquicos involucrados en la dirección y ejecución de proyectos TI. Los resultados de la entrevista se contrastaron con los principales desafíos levantados a nivel nacional con base en una encuesta, construida a partir de las entrevistas, la cual fue enviada a profesionales que se encuentran trabajando en áreas TI.

\section{Población sobre la que se efectúa el estudio}

Se utilizó la empresa de TI Ecofor Servicios Tecnológicos Limitada, incluyendo personal relacionado con la gestión y la ejecución de proyectos de software de las áreas gerencial, electrónica y de desarrollo. Se seleccionaron gerentes en un $27 \%$, ingenieros de proyecto en un $18 \%$ y analistas programadores en un 55\%. En total participaron 11 
profesionales con una edad promedio de 38 años y un mínimo de 27 y un máximo de 58. Poseían en promedio 8 años de servicio en la compañía con un mínimo de 2 y un máximo de 20. De ellos un $42 \%$ ha trabajado en otras compañías TI.

\section{Entorno}

El estudio se efectuó en la empresa Ecofor Servicios Tecnológicos que cuenta con 25 trabajadores y nació en 2004 como una compañía TI con el objetivo ayudar a sus clientes a superar retos en la industria forestal, agropecuaria, comercial y portuaria a través de soluciones compuestas por desarrollo de software a medida, tanto móvil como web, desarrollo de hardware, sistemas embebidos y asesoría tecnológica, buscando mejorar los procesos y la productividad de empresas líderes a nivel nacional como CMPC, Forestal Mininco, Celulosa Arauco y Puerto Lirquén.

El negocio de la empresa se divide en tres líneas principales, una de ellas son productos electrónicos embebidos que solucionen las necesidades comunes de empresas de diversos rubros, otra son productos de software que se comercializan con un costo mensual para el cliente y finalmente sistemas a medida que pueden realizarse para el cliente interno Ecofor Forestal o para clientes externos.

\section{Intervenciones}

Antes de comenzar la entrevista se introdujo a los participantes en el contexto de esta, con la finalidad de evitar respuestas fuera del alcance del estudio, respecto a la agilidad todos contaban con un grado de conocimiento, ya que en el pasado se intentó implementar la metodología en la organización sin obtener buenos resultados. Para realizar las entrevistas se utilizó como medio de recolección de datos un dispositivo para grabar audio y un documento escrito con las preguntas a realizar, el cual fue completado por el entrevistador. Para los grupos de entrevista y discusión, la grabación de voz fue un apoyo para complementar mediante preguntas abiertas las preguntas base de la entrevista. Simultáneamente, el investigador Ilevó un diario de campo donde registró cada una de las situaciones previstas para la recolección de información y las percepciones del contexto empresarial durante las entrevistas realizadas. Las etapas de la entrevista se dividieron en caracterización del presente y comprensión de la realidad, propuesta de alto impacto y alertas sobre las transformaciones, considerando 13 preguntas base.

Al terminar las entrevistas, se compartió una encuesta de 11 preguntas dirigida a profesionales externos a la compañía y ligados al área $\mathrm{TI}$, con el fin de conocer la realidad de otras organizaciones nacionales respecto a la adopción de metodologías ágiles y contrastarla con los resultados obtenidos en las entrevistas. Las preguntas se originaron con base en las respuestas de las entrevistas, la cuales fueron:

- ¿Cuál es la principal metodología que utiliza su empresa?

- ¿Hace cuánto tiempo su empresa utiliza esta metodología?

- ¿Qué tan de acuerdo está con la metodología de gestión de proyectos de su organización?

- ¿ ¿Ha participado en proyectos ágiles?

- ¿Cuál de estas frases define de mejor manera lo que involucra una metodología ágil?

- ¿Qué tan importante es para usted la gestión de proyectos bajo metodología ágil en una organización?

- De acuerdo con su criterio, ordene de 1 a 10 (siendo 1 el menos importante y 10 el más importante) los siguientes desafíos para implementar metodología ágil en una organización (cultura organizacional en desacuerdo con los valores ágiles, resistencia al cambio, falta de compromiso de la gerencia, falta de habilidades o experiencia con metodologías ágiles, capacitación insuficiente, procesos y prácticas inconsistentes entre equipos, falta de compromiso del cliente, metodología tradicional 
arraigada en la organización, naturaleza de los proyectos, realizar cambios contractuales).

- De acuerdo con su criterio, ordene de 1 a 11 (siendo 1 el menos importante y 11 el más importante) los siguientes beneficios de adoptar metodología ágil en una organización (acelerar la entrega de software, mejorar la capacidad de gestionar prioridades cambiantes, aumentar la productividad, mejorar la comunicación con el cliente, mejorar la calidad del software, mejorar la previsibilidad de la entrega, reducir el costo del proyecto, mejorar la moral del equipo, reducir el riesgo del proyecto, aumentar la mantenibilidad del software, gestionar mejor los equipos distribuidos).

- De acuerdo con su criterio, ordene de 1 a 6 (siendo 1 el menos importante y 6 el más importante) los siguientes costos de adoptar metodología ágil en una organización (capacitación, tiempo de adaptación, pérdida de clientes, desvinculación de personal, reestructuración de puestos de trabajo, sobrepresupuesto en los proyectos iniciales).

- ¿Cuál sería la principal acción que adoptaría para lograr una transición de una metodología tradicional a una ágil?

- ¿Cuál es la principal amenaza que detecta respecto a la implementación de una metodología ágil?

Para registrar las respuestas se utilizó la plataforma de encuestas en línea Formularios de Google, la que permitió elaborar la encuesta y efectuarla de forma independiente por profesionales vinculados a organizaciones con áreas TI.

\section{Método de verificación y validación del instrumento}

Con el objetivo de corroborar el instrumento, este fue analizado y verificado por tres expertos chilenos con grado académico de doctor, quienes indicaron sugerencias y ajustes de acuerdo con el diseño de la investigación. En relación con la validación de la información obtenida, se consideraron los comentarios de pares y comprobaciones con los participantes, tomando en cuenta este criterio se compartieron los resultados y las interpretaciones con los actores clave, quienes en su calidad de participantes del estudio han permitido matizar las interpretaciones y reflexiones que han surgido del total de los datos analizados.

\section{Plan de análisis de los datos}

Se confeccionó el instrumento y sus preguntas estableciendo las categorías de observación y estudio. Luego se concretaron entrevistas (11) con diversos perfiles de las áreas empresariales de la organización y se realizó una observación (no participante). Posteriormente se realizó un contraste entre la teoría y las observaciones recogidas tomando nota de estas en un diario de campo y se plantearon hipótesis explicativas sobre dichas observaciones. Con la información obtenida se realizó un análisis de las percepciones con el fin elaborar una encuesta a profesionales TI (29) que permitiera contrastar la realidad de la empresa de estudio con otras a nivel nacional.

\section{Ética}

Se procuró la participación informada y voluntaria de los actores del estudio. Además se tomaron medidas para garantizar la confidencialidad de la información y validarla con los propios interesados (por ejemplo, cambio de nombres que no necesariamente coinciden con su género). No se intentó, ni implícita ni explícitamente, influir en las respuestas, ni cambiar sus características individuales, se respetó la libertad de participar o no sin presión, ni ofrecimiento de beneficio para ser parte de la investigación.

\section{Resultados}

Para el análisis de resultados se ha realizado una categorización mediante un proceso deductivo, 
extrayendo los criterios que permiten entender la problemática desde los actores clave. Además se ha puesto atención a situaciones que podrían surgir de las entrevistas y de las observaciones efectuadas. Esta categorización es una selección de factores y elementos para considerar, estando conscientes de que se podría diversificar aún más por la amplitud del constructo, la problemática abordada y su práctica. Los datos se muestran agrupados de acuerdo con la tabla de categorización (Tabla 1).

\section{Discusión de resultados}

Los resultados del estudio respecto a la caracterización del presente y la comprensión de la realidad indican que la mayor parte de la organización no comprende el procedimiento de trabajo actual, generando prácticas inconsistentes entre las distintas áreas, siendo un aspecto relevante de acuerdo con el 35\%. Además, mediante la observación prolongada se confirma lo expuesto en la entrevista, en la que gran parte de la capa estratégica y operativa trabaja con una alta improvisación y ausencia de control, lo cual es un desafío que enfrentan las organizaciones durante la etapa de adopción (Gandomani et al., 2015). Igualmente, esta falta de conocimiento en la organización respecto a los procesos actuales puede ocasionar que la transición fracase (Landim, Albuquerque y Macedo 2010). Asimismo, la cultura organizacional actual puede influir negativamente en la aceptación de los roles y responsabilidades ágiles como aclaran Nerur, Mahapatra y Mangalaraj (2005), ya que la orientación organizacional es burocrática con una jerarquía estricta. Esto explica que la metodología actual nunca haya entrado en un proceso de mejora continua para ser adaptada a la realidad de la organización, creando malas prácticas y falta de definiciones contractuales, impactando directamente en los presupuestos, debido a la falta de adaptabilidad ante cambios de los clientes; a pesar de ello, el 51\% de los encuestados reconoce que esta falta de involucramiento del cliente es una barrera a superar para lograr una transición exitosa (Hoda, Noble y Marshall, 2011) y que es necesario realizar cambios contractuales con el cliente como indica el $42 \%$ de los encuestados y fundamentan en su estudio Hoda, Noble y Marshall (2009). Por lo tanto, los procesos comerciales actuales difieren de las prácticas ágiles, ya que necesitan que los requerimientos estén definidos desde el comienzo del proyecto y los pagos se acuerden mediante la finalización de hitos.

Respecto a la resistencia que es común en cualquier proceso de cambio, el $49 \%$ de los encuestados indica que es una brecha por superar (Gandomani et al., 2015). Como consecuencia de lo anterior, la organización no ha visto en la agilidad una herramienta para mejorar sus problemas.

Desde los hallazgos que considera la propuesta de alto impacto se evidencia que la organización presenta falta de habilidades o de experiencia con métodos ágiles, siendo esto una brecha relevante como indica el 50\% y establecen Solinski y Petersen (2014), por lo cual no se han logrado ver claramente sus principales beneficios, en contraste con los perfiles encuestados, quienes señalan como beneficios directos de la agilidad el poder gestionar prioridades cambiantes y acelerar la entrega del software. Esta falta de conocimiento en la capa estratégica puede ocasionar gestión y patrocinio inadecuados respecto a la adopción de la agilidad tal como lo indica el 54\% y el estudio de Chow y Cao (2008), además de crear una cultura en desacuerdo con los valores ágiles como lo evidencia el 59\% y Tolfo et al. (2011). La observación prolongada entrega como respuesta a este hallazgo el hecho de que parte de los perfiles estratégicos carecen de competencias TI debido a su orientación comercial y a que los perfiles TI no se han mantenido constantemente actualizados respecto a las tendencias, coincidiendo con lo expuesto por Moe, Aurum y Dybå (2012), que evidencian que los principales problemas con la alineación entre los niveles estratégicos, operacionales y tácticos es la falta de comprensión compartida y el entendimiento de la complejidad de las tareas. 
Tabla I: Categorización.

\begin{tabular}{|c|c|}
\hline Ítem & Categorías \\
\hline $\begin{array}{l}\text { En la práctica ¿cómo opera hoy la gestión de proyectos } \\
\text { en la empresa? }\end{array}$ & $\begin{array}{l}\text { - Mediante una autogestión que no contempla metodología } \\
\text { - } \quad \text { Mediante una metodología tradicional } \\
\quad \text { Desconoce el procedimiento }\end{array}$ \\
\hline $\begin{array}{l}\text { ¿Cómo opera la gestión de proyectos de cara a los } \\
\text { clientes? }\end{array}$ & $\begin{array}{ll}-\quad & \text { Depende del tipo de cliente (interno/externo) } \\
\text { - } \quad \text { No gestiona directamente con clientes } \\
\text { - } \quad \text { Otra (defina) }\end{array}$ \\
\hline $\begin{array}{l}\text { ¿Cómo calificaría usted la metodología de gestión de } \\
\text { proyectos actual? }\end{array}$ & $\begin{array}{l}\text { - } \quad \text { Es algo irrelevante para el cliente } \\
\text { - } \quad \text { Cumple co adapta a la realidad de la organización } \\
\quad \text { Cumplativas }\end{array}$ \\
\hline $\begin{array}{l}\text { ¿De dónde surge la necesidad de un cambio de } \\
\text { metodología? }\end{array}$ & $\begin{array}{ll}\text { - } & \text { Mayor rendimiento del equipo } \\
\text { - } \quad \text { Realizar tareas bajo un estándar definido } \\
\text { - } \quad \text { Para lograrar la integración entientre áreas } \\
\text { Para cumplir con el resultado esperado por el cliente }\end{array}$ \\
\hline ¿Qué es para usted la agilidad? & $\begin{array}{l}\text { - } \quad \text { Es una metodología para compañías grandes } \\
\text { - } \quad \text { Producir más en menos tiempo } \\
\text { Poder adaptarse al cambio }\end{array}$ \\
\hline $\begin{array}{l}\text { ¿Cómo entiende usted el proceso de transición de una } \\
\text { metodología tradicional a una ágil? }\end{array}$ & $\begin{array}{ll}\text { - } & \text { Apoyado por un acuerdo transversal a la organización } \\
\text { - } \quad \text { Apoyado con perfiles versátiles y roles claramente definidos } \\
\text { - } \quad \text { Apoyado con un plan de capacitampión transversal } \\
\text { - No logra especificar cómo llevar a cabo la transición } \\
\quad \text { Otra (defina) }\end{array}$ \\
\hline $\begin{array}{l}\text { ¿Tiene propuestas específicas de mejora o cambio de } \\
\text { corto plazo y alto impacto? }\end{array}$ & $\begin{array}{ll}- & \text { Plan de capacitación } \\
\text { - } \quad \text { Aumentar la frecuencia de presentación de avances a clientes } \\
\text { - } \quad \text { Integrar la comunicación interna } \\
\quad \text { Otra (defina) }\end{array}$ \\
\hline $\begin{array}{l}\text { Si yo le propusiera una estrategia de cambio basada en } \\
\text { un análisis de la percepción de los diferentes niveles } \\
\text { jerárquicos de la compañía frente a una futura adopción } \\
\text { ágil como método para la gestión de proyectos, ¿qué } \\
\text { opina de dicha propuesta o del cambio planteado? }\end{array}$ & $\begin{array}{l}\text { - Un buen punto de partida para evidenciar brechas } \\
\text { - } \quad \text { Levantar deficiencias que no permiten adoptar las tendencias del mercado } \\
\text { - Necesario para alinear la forma de trabajo en la organización } \\
\text { implementar la metododología personas y recursos son los adecuados para }\end{array}$ \\
\hline $\begin{array}{l}\text { En su opinión, ¿cuáles son los costos de implementar } \\
\text { metodologías ágiles para la empresa? }\end{array}$ & $\begin{array}{ll}- & \text { No existen costos } \\
\text { - } \quad \text { Capacitación } \\
\text { - } \quad \text { Tiempo de adaptación } \\
\text { Otra (defina) }\end{array}$ \\
\hline $\begin{array}{l}\text { ¿Qué tipo de amenaza o riesgo podría provocar en la } \\
\text { organización un plan de transición a ágil? }\end{array}$ & $\begin{array}{ll}\text { - } & \text { No veo ninguna amenaza o riesgo } \\
\text { - } \quad \text { Folta de convencimiento de la alta gerencia } \\
\text { - } \quad \text { Resistencia al cambio } \\
\text { - } \quad \text { Que algunos que existirán problemas al inicio } \\
\quad \quad \text { Que la capa gerencial subestime el tiempo para obtener resultados }\end{array}$ \\
\hline $\begin{array}{l}\text { ¿Cuáles son los factores que facilitarían en esta empresa } \\
\text { la implementación de metodologías ágiles? }\end{array}$ & $\begin{array}{l}\text { - } \quad \text { La buena disposición de la capa operativa } \\
\text { - } \quad \text { Contar con un equipo reducido } \\
\text { - } \quad \text { No veo factores que facilitarían la implementación } \\
\quad \text { Otra (defina) }\end{array}$ \\
\hline $\begin{array}{l}\text { ¿Cuáles son los factores que obstaculizarían en esta } \\
\text { empresa, la implementación de metodologías ágiles? }\end{array}$ & $\begin{array}{ll} & \text { Falta de habilidades blandas en el equipo } \\
\text { - } & \text { Que la alta dirección lo vea como gasto innecesario } \\
\text { - } & \text { Metodo especialización } \\
& \text { Resistencia al cambional muy adherida en la cultura organizacional }\end{array}$ \\
\hline $\begin{array}{l}\text { ¿Cuál sería su principal preocupación respecto a la } \\
\text { aplicación o implementación de metodología ágil? }\end{array}$ & $\begin{array}{l}\text { - } \quad \text { Una correcta implementación } \\
\text { - } \quad \text { La etapa inicial de adopción } \\
\quad \text { Que no resulte }\end{array}$ \\
\hline
\end{tabular}

Fuente: Elaboración propia. 
Kanavittaya, Armarego y Goulding (2010) establecen que en las PyMEs las decisiones las toma mayormente el propietario mostrando poca preocupación por la planificación estratégica TI. De igual manera, la observación prolongada demuestra que la alta gerencia y la subgerencia no quieren renunciar a su autoridad, dificultando la transición (Nerur, Mahapatra y Mangalaraj, 2005). Lo anterior explica por qué no se ha logrado mejorar la gestión de proyectos anteriormente, ya que los cambios siempre surgen de la capa operativa sin un mayor involucramiento y compromiso de la capa estratégica.

Es preocupante que en la etapa de alerta sobre las transformaciones, la capa estratégica no vincule los costos asociados a la adopción de la agilidad, ya que a pesar de sus múltiples beneficios esta involucra costos directos como el de capacitación, tal como indica el $40 \%$ señalando que es una brecha relevante, pues al ser insuficiente dificulta la adopción, lo cual coincide con el estudio de Gandomani et al. (2014). En esta misma línea, Gandomani y Nafchi (2016) manifiestan que tener expectativas equivocadas sobre la agilidad es un riesgo importante en la etapa de adopción y que las expectativas de los gerentes respecto a los métodos y valores ágiles pueden estar completamente equivocadas, creando nuevos desafíos; además, la falta de comunicación puede aumentar aún más las brechas existentes, como indican el 55\% de los entrevistados y Srinivasan y Lundqvist (2010). Adicionalmente, los entrevistados señalan como principal riesgo la falta de conocimiento de la gerencia respecto a los beneficios de la agilidad, producto de una cultura organizacional fuertemente orientada a las metodologías tradicionales, como indica el $54 \%$ de los encuestados, explicando la falta de patrocinio de la alta gerencia que coincide con la observación prolongada y la opinión de los encuestados respecto a las principales amenazas en la adopción. A pesar de las dificultades detectadas, los entrevistados coinciden en la buena disposición de la capa operativa en cambiar su proceso de gestión por uno ágil; sin embargo, manifiestan preocupación respecto del periodo de implementación, producto de que la organización no contemple aspectos necesarios para una correcta transición tales como subestimar el tiempo para la entrega de resultados o la falta de proyectos piloto.

\section{Conclusiones}

Este trabajo muestra que es posible implementar metodologías ágiles para gestionar sus proyectos en una PyME TI con metodología tradicional. Lo que se evidencia mediante el análisis de percepciones de los integrantes de la empresa en estudio. Los resultados muestran que el $90 \%$ de los entrevistados logra manifestar diversos motivos que fundamentan una adopción ágil, de ellos, un 73\% indica que el principal es la falta de adaptación de la metodología actual a la realidad de la organización, debido a su estructura rígida y a las múltiples fases exigidas a lo largo del proceso, lo cual contrasta con las metodologías ágiles.

En este sentido, se entiende el proceso de adopción como un acuerdo transversal apoyado por la alta gerencia para alinear la forma de trabajo con una metodología que permita a los proyectos adaptarse a entornos cambiantes, aunque no sin antes trabajar en aspectos como la resistencia al cambio, una cultura organizacional que sustente los principios ágiles y la asesoría de profesionales expertos para lograr una implementación adecuada.

Los hallazgos indican que la propuesta de esta investigación es bien recibida y que contribuye a identificar las principales brechas a las que una organización con metodología tradicional se ve expuesta al momento de implementar metodologías ágiles para la gestión de proyectos, permitiendo abordarlas antes de comenzar la etapa de transición.

En este contexto, el plan de acción que se debe llevar a cabo para abordar dichas brechas contempla como punto de partida transparentar a la alta gerencia las principales etapas de la adopción, así como sus costos, plazos y riesgos, comparándolos con los beneficios a corto y mediano 
plazo, con el fin de disminuir el grado de incertidumbre y asegurar su compromiso durante toda la etapa de adopción. Posterior a esta etapa, se propone trabajar en un plan de cambio de cultura organizacional que se encuentre alineado con los principios ágiles y que promueva el mejoramiento continuo y la comunicación, facilitando la posterior implementación de la agilidad. Esta medida debe acompañarse además por un análisis de competencias de los diversos cargos de la organización con el fin de determinar si cumplen con los perfiles requeridos para adaptarse al cambio metodológico, lo cual involucra un plan de capacitación transversal que permita nivelar la capa estratégica con la operativa, otorgando herramientas que permitan adoptar la agilidad a nivel transversal.

En paralelo a las medidas mencionadas, se propone trabajar en la definición de contratos ágiles que determinen las responsabilidades de todos los involucrados dentro de un proyecto, así como la participación del cliente durante cada etapa con la finalidad de garantizar una correcta implementación de la agilidad.

Por otra parte, se sugiere apoyar las etapas iniciales de la metodología mediante expertos que faciliten la adopción de los principios y las prácticas ágiles a los líderes de equipo y que además analicen estas prácticas y detecten si se alinean completamente al contexto de la empresa o si se deben adaptar, con el fin de incluir solo las que aporten valor al negocio, para luego implementar proyectos piloto que disminuyan la incertidumbre y permitan a la organización poner en práctica la metodología de forma controlada.

\section{Agradecimientos}

Los autores agradecen al programa de Magister en Ingeniería Industrial y de Sistemas de la Universidad del Desarrollo de Chile. También, se agradece a la Facultad de Ingeniería y Vicerrectoría de Investigación y Doctorados de la Universidad Autónoma de Chile, por el apoyo otorgado.

\section{Referencias}

Beck, K. et al. (2001). Manifesto for Agile Software Development. http://agilemanifesto.org/

Chow, T., Cao, D. (2008). A survey study of critical success factors in agile software projects. Journal of Systems and Software, 81(6), 961-971. https://doi. org/10.1016/j.jss.2007.08.020

CollabNet VersionOne. (2019). $13^{\text {th }}$ Annual State of Agile Report. https://stateofagile.com/\#ufh-i-61355341813th-annual-state-of-agile-report/7027494

Collyer, S., Warren, C., Hemsley, B., Stevens, C. (2010). Aim, fire, aim - project planning styles in dynamic environments. Project Management Journal, 41(4), 108-121. https://doi.org/10.1002/pmj.20199

de Souza Bermejo, P. H., Zambalde, A. L., ToneIli, A. O., Souza, S. A., Zuppo, L. A., Rosa, P. L. (2014). Agile principles and achievement of success in software development: A quantitative study in Brazilian organizations. Procedia Technology, 16, 718-727. https://doi.org/10.1016/j. protcy.2014.10.021

Gandomani, T., Nafchi, M. Z. (2016). Agile transition and adoption human-related challenges and issues: A grounded theory approach. Computers in Human Behavior, 62, 257-266. https://doi.org/10.1016/j. chb.2016.04.009

Gandomani, T. J., Zulzalil, H., Ghani, A., Sultan, A. B., Parizi, R. M. (2015). The impact of inadequate and dysfunctional training on agile transformation process: A grounded theory study. Information and Software Technology, 57, 295-309. https://doi.org/10.1016/j.infsof.2014.05.011

Gandomani, T. J., Zulzalil, H., Ghani, A., Sultan, A. B., Sharif, K. Y. (2014). How human aspects impress agile software development transition and adoption. International Journal of Software Engineering and Its Applications, 8(1), 129-148

Göksu, M. (2018). Comparison of the Agile Methodologies and the Waterfall [Tesis de doctorado], Near East University, Turquía

Hoda, R., Noble, J., Marshall, S. (2011). The impact of inadequate customer collaboration on self-organizing agile teams. Information and 
Software Technology, 53(5), 521-534. https://doi. org/10.1016/j.infsof.2010.10.009

Hoda, R., Noble, J., Marshall, S. (2009). Negotiating contracts for agile projects: A practical perspective. Lecture Notes in Business Information Processing, 31, 186-191. https://doi. org/10.1007/978-3-642-01853-4_25

Hohl, P., Klünder, J., van Bennekum, A., Lockard, R., Gifford, J., Münch, J., Stupperich, M., Schneider, K. (2018). Back to the future: Origins and directions of the "Agile Manifesto" - views of the originators. Journal of Software Engineering Research and Development, 6(1), e15. https://doi.org/10.1186/ s40411-018-0059-z

Kanavittaya, P., Armarego, J., Goulding, P. (2010). The alignment of business strategy with agile software development within SMEs. En P. Bharati, I. Lee, \& A. Chaudhury (Eds.), Global Perspectives on SmaII and Medium Enterprises and Strategic Information Systems: International Approaches, 215-233. https://doi.org/10.4018/978-1-61520-627-8.ch011

Klünder, J., Schmitt, A., Hohl, P., Schneider, K. (2017). Fake news: Simply agile. En A. Volland, M. Engstler, M. Fazal-Baqaie, E. Hanser, O. Linssen, M. Mikusz, (Eds.), Projektmanagement und Vorgehensmodelle 2017 - Die Spannung zwischen dem Prozess und den Menschen im Projekt (pp. 187-192). Bonn: Gesellschaft für Informatik

Landim, H. F., Albuquerque, A. B., Macedo, T. C. (2010). Procedures and conditions that influence on the efficiency of some agile practices. En: Seventh International Conference on the Quality of Information and Communications Technology (QUATIC). https://doi.org/10.1109/quatic.2010.70

Moe, N. B., Aurum, A., Dybå, T. (2012). Challenges of shared decision-making: A multiple case study of agile software development. Information and Software Technology, 54(8), 853-865. https://doi.org/10.1016/j.infsof.2011.11.006

Mohallel, A. A., Bass, J. M. (2019). Agile software development practices in Egypt SMEs: A grounded theory investigation. IFIP International Federation for Information Processing, 551, 355-365. https:// doi.org/10.1007/978-3-030-18400-1_29
Nerur, S., Mahapatra, R., Mangalaraj, G. (2005). ChaIlenges of migrating to agile methodologies. Communications of the ACM, 48(5), 72-78. https://doi. org/10.1145/1060710.1060712

Nishijima, R. T., Dos Santos, J. G. (2013). The challenge of implementing scrum agile methodology in a traditional development environment. International Journal of Computers \& Technology, 5(2), 98-108. https://doi.org/10.24297/ijct.v5i2.3529

Patanakul, P., Henry, J., Leach, J. A. (2015). Agile project execution. En R. J. Martinelli, \& D. Z. Milosevic, Project Management ToolBox (pp. 301-322). https://doi.org/10.1002/9781119174820.ch11

Pikkarainen, M., Salo, O., Kuusela, R., Abrahamsson, P. (2012). Strengths and barriers behind the successful agile deployment-insights from the three software intensive companies in Finland. Empirical Software Engineering, 17(6), 675-702. https://doi. org/10.1007/s10664-011-9185-5

PMI. (2018). Success in Disruptive Time: Expanding the Value Delivery Landscape to Address the High Cost of Low Performance. Project Management Institute. https://www.pmi.org/-/media/pmi/documents/ public/pdf/learning/thought-leadership/pulse/pulse-of-the-profession-2018.pdf

Rodríguez, D., Valldeoriola, J. (2009). Metodología de la investigación. Universitat Oberta de Catalunya. http://openaccess.uoc.edu/webapps/o2/bitstream/10609/77608/2/Metodolog\%C3\%ADa\%20 de\%20la\%20investigaci\%C3\%B3n_M\%C3\%B3dulo\%201.pdf

Senapathi, M., Srinivasan, A. (2012). Understanding post-adoptive agile usage: An exploratory cross-case analysis. Journal of Systems and Software, 85(6), 1255-1268. https://doi.org/10.1016/j. jss.2012.02.025

Solinski, A., Petersen, K. (2014). Prioritizing agile benefits and limitations in relation to practice usage. Software Quality Journal, 24(2), 447-482. https://doi. org/10.1007/s11219-014-9253-3

Srinivasan, J., Lundqvist, K. (2010). Agile in India: chaIlenges and lessons learned. En: Proceedings of the $3^{\text {rd }}$ India software engineering conference. https:// doi.org/10.1145/1730874.1730898 
Tolfo, C., Wazlawick, R. S., Gomes Ferreira, M. G., Forcellini, F. A. (2011). Agile methods and organizational culture: Reflections about cultural levels. Journal of Software Maintenance and Evolution: Research and Practice, 23(6), 423-441. https://doi. org/10.1002/smr.483
Yepes González, J. D., Pardo Calvache, C. J., Gómez Gómez, O. S. (2015). Revisión sistemática acerca de la implementación de metodologías ágiles y otros modelos en micro, pequeñas y medianas empresas de software. Revista Tecnológica ESPOL, 28(5), 464-479 